\title{
Reply to Rizzatti et al.
}

We would like to thank Dr. Rizzatti and colleagues for their interest in our paper entitled "Endoscopic ultrasound and fine-needle aspiration for the detection of residual nodal disease after neoadjuvant chemoradiotherapy for esophageal cancer" [1].

In their letter to the editor, Dr. Rizzatti and colleagues stress the need for standardization of restaging strategies to improve the detection rate of residual nodal disease after neoadjuvant chemoradiotherapy for esophageal cancer. The authors suggest a systematic approach in which sampling of an adjacent lymph node (LN) station is only performed in the absence of a positive smear from the previously sampled LN station - comparable to an algorithm that was previously published on initial staging of esophageal cancer [2].

We agree that a change of diagnostic strategy is needed in this clinical setting. After neoadjuvant chemoradiotherapy, residual nodal disease cannot reliably be ruled out based on endoscopic ultrasound (EUS) features alone, necessitating concomitant fine-needle aspiration (FNA) sampling, preferably in the presence of rapid on-site cytopathological evaluation (ROSE). However, we believe that, even in the presence of ROSE, adequate sampling of LNs will remain challenging owing to neoadjuvant chemoradiotherapy-induced fibrosis and the focal distribution of vital tumor cells [3]. Indeed, development of a restaging algorithm may be an important step forward.
Ideally, such a restaging algorithm should take into account LN distribution based on both patient and disease characteristics, and enable targeting of the LNs that are most likely to be affected. The results of the ongoing TIGER study a study on the LN distribution in resectable esophageal cancer after neoadjuvant therapy - may serve to develop such tool [4].

\section{Competing interests}

The authors declare that they have no conflict of interest.

The authors

Ruben D. van der Bogt ${ }^{1}$, Berend J. van der Wilk $^{2}$, Jan J. B. van Lanschot ${ }^{2}$, Manon C. W. Spaander ${ }^{1}$

1 Department of Gastroenterology and Hepatology, Erasmus MC University Medical Center, Rotterdam, The Netherlands

2 Department of Surgery, Erasmus MC University Medical Center, Rotterdam, The Netherlands

\section{Corresponding author}

\section{C. W. Spaander, MD}

Erasmus MC University Medical Center, Doctor Molewaterplein 40, 3015 GD, Rotterdam, The Netherlands v.spaander@erasmusmc.nl

\section{References}

[1] van der Bogt RD, van der Wilk BJ, Poley JW et al. Endoscopic ultrasound and fine-needle aspiration for the detection of residual nodal disease after neoadjuvant chemoradiotherapy for esophageal cancer. Endoscopy 2019: doi:10.1055/a-1065-1759

[2] Vazquez-Sequeiros E, Wiersema M], Clain JE et al. Impact of lymph node staging on therapy of esophageal carcinoma. Gastroenterology 2003; 125: 1626-1635

[3] Zuccaro G]r., Rice TW, Goldblum J et al. Endoscopic ultrasound cannot determine suitability for esophagectomy after aggressive chemoradiotherapy for esophageal cancer. Am J Gastroenterol 1999; 94: $906-$ 912

[4] Hagens ERC, van Berge Henegouwen MI, van Sandick JW et al. Distribution of lymph node metastases in esophageal carcinoma [TIGER study]: study protocol of a multinational observational study. BMC Cancer 2019; 19 : 662

Bibliography

DOI https://doi.org/10.1055/a-1114-2608

Endoscopy 2020; 52: 317

(c) Georg Thieme Verlag KG

Stuttgart · New York

ISSN 0013-726X 